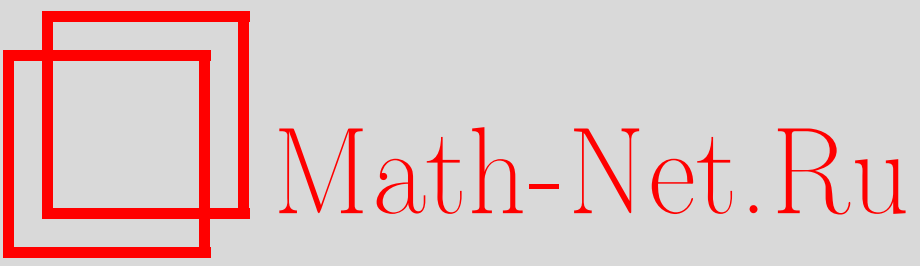

П. В. Ролдугин, Об одном методе построения булевых функций малого веса, не имеющих имплицент от фиксированного числа переменных, Матем. вопр. криптогр., 2016, том 7, выпуск 3, 73-92

DOI: https://doi.org/10.4213/mvk197

Использование Общероссийского математического портала Math-Net.Ru подразумевает, что вы прочитали и согласны с пользовательским соглашением

http://www. mathnet.ru/rus/agreement

Параметры загрузки:

IP : 54.237 .206 .68

26 апреля 2023 г., 14:13:49 
МАТЕМАТИЧЕСКИЕ ВОПРОСЫ КРИПТОГРАФИИ

2016 T. 7 № 3 C. $73-92$

УДК 519.719.2+519.712

\title{
Об одном методе построения булевых функций малого веса, не имеющих имплицент от фиксированного числа переменных
}

\author{
П. В. Ролдугин
}

Московский государственный университет информационных технологий, радиотехники и электроники, Москва

Получено 20.IV.2014

Аннотация. Задача построения булевых функций без имплицент от $k$ переменных сведена к построению такого множества $M$ булевых функций от $k-1$, что для любых различных векторов $\bar{\beta}_{1}, \ldots, \bar{\beta}_{k} \in V_{k-1}$ и любых $\alpha_{1}, \ldots, \alpha_{k} \in\{0,1\}$ существует $f \in M: f\left(\bar{\beta}_{1}\right)=\alpha_{1}, \ldots, f\left(\bar{\beta}_{k}\right)=\alpha_{k}$. Это позволяет строить функции без имплицент от $k$ переменных, имеющие вес, близкий к минимально возможному значению. Построено несколько семейств таких булевых функций.

Ключевые слова: булевы функции, имплиценты, двоичные матрицы

On a method for constructing low-weight Boolean functions without majorants of the given number of variables

\section{P. V. Roldugin}

Moscow State University of Information Technologies, Radioengineering and Electronics, Moscow

Abstract. The problem of constructing Boolean functions without majorants of $k$ variables is reduced to the construction of a set $M$ of Boolean functions of $k-1$ variables such that for any different vectors $\bar{\beta}_{1}, \ldots, \bar{\beta}_{k} \in V_{k-1}$ and for any $a_{1}, \ldots, a_{k} \in\{0,1\}$ there exists a function $f \in M: f\left(\bar{\beta}_{1}\right)=a_{1}, \ldots, f\left(\bar{\beta}_{k}\right)=a_{k}$. This approach permits to construct functions $f$ of small weight having no $k$ variable majorants. Several families of such Boolean functions $f$ are constructed.

Key words: Boolean functions, majorants, Boolean matrices

Citation: Mathematical Aspects of Cryptography, 2016, v. 7, № 3, pp. 73-92 (Russian)

(c) Академия криптографии Российской Федерации, 2016 г. 


\section{Введение}

Множество всех булевых функций от $\mathrm{n}$ переменных обозначим через $F_{n}$, пространство $k$-мерных векторов над $G F(2)$ - через $V_{k}$, множество выполняющих векторов функции $f\left(x_{1}, \ldots, x_{n}\right)$ - через $E_{f}$. Число переменных функции будем называть также ее длиной.

Настоящая работа является продолжением исследований ([1-3]) класса булевых функций, не имеющих имплицент от фиксированного числа переменных. Напомним ([6]), что имплицентой булевой функции $f\left(x_{1}, \ldots, x_{n}\right)$ называется такая булева функция $g\left(x_{i_{1}}, \ldots, x_{i_{k}}\right)$, что $f\left(x_{1}, \ldots, x_{n}\right)$. $g\left(x_{i_{1}}, \ldots, x_{i_{k}}\right)=f\left(x_{1}, \ldots, x_{n}\right)$ для любых значений аргументов $x_{1}, \ldots, x_{n}$. Обозначим через $G_{n}^{(k)} \subset F_{n}$ множество булевых функций от $n$ переменных, не имеющих имплицент длины $k$ или меньше. Далее считаем, что $n>k$. У отличной от константы функции от $n$ переменных всегда есть имплицента длины $n$ - это она сама. Кроме того, случай $k=1$ несложен и полностью разобран в [2], поэтому везде далее предполагаем $k \geqslant 2$.

Отметим одно из возможных применений исследуемых имплицент. Допустим, что необходимо проверить совместность системы булевых уравнений $\left\{f\left(q_{i}\left(x_{1}, \ldots, x_{n}\right)\right)=1, i=1,2, \ldots\right\}$, где $f: V_{n} \rightarrow V_{1}, q_{i}: V_{n} \rightarrow V_{n}$. Если у функции $f\left(x_{1}, \ldots, x_{n}\right)$ есть имплицента $g\left(x_{i_{1}}, \ldots, x_{i_{k}}\right)$, где $k<n$, то из несовместности системы $\left\{g\left(q_{i}\left(x_{1}, \ldots, x_{n}\right)\right)=1, i=1,2, \ldots\right\}$ следует несовместность исходной системы. Вторая система может допускать более простой способ проверки совместности за счет того, что каждое уравнение зависит не от $n$, а не более чем от $k$ переменных. Системы уравнений, в которых $f \in G_{n}^{(k)}$, заведомо не допускают подобного сведения.

В [2] доказана теорема, которая позволяет представлять функции из $G_{n}^{(k)}$ как двоичные матрицы со свойством комбинаторной полноты порядка $k$. Более подробно: двоичная матрица $A$ размера $m \times n$ называется комбинаторно полной порядка $k$, если для любого набора $1 \leqslant i_{1}<\cdots<i_{k} \leqslant n$ матрица, составленная из столбцов матрицы $A$ с номерами $i_{1}, \ldots, i_{k}$, содержит все $2^{k}$ возможных векторов из $V_{k}$ в качестве строк.

Теорема 1 ([2]). Булева функция $f$, отличная от константы, не имеет имплицент от $k$ переменных тогда и только тогда, когда матрица, составленная из векторов множества $E_{f}$, является комбинаторно полной порядка $k$.

Отметим, что перестановка строк комбинаторно полной матрицы дает комбинаторно полную матрицу того же порядка, поэтому при составлении матрицы из векторов множества $E_{f}$ порядок перечисления векторов не важен. 
На основе теоремы 1 далее вместо методов построения функции $f \in G_{n}^{(k)}$ приводятся методы построения комбинаторно полной матрицы порядка $k$; при этом вес функции $f$ есть число строк этой матрицы, если она не содержит одинаковых строк.

Рассмотрим величину $m_{\min }^{(k)}(n)-$ минимально возможное число строк комбинаторно полной матрицы порядка $k$ с $n$ столбцами. Из теоремы 1 следует, что $m_{\min }^{(k)}(n)=\min _{f \in G_{n}^{(k)}}\|f\|$, где $\|f\|-$ вес функции $f$. В [1-3] доказаны следующие оценки: $2^{k} \cdot k \cdot \ln n \geqslant m_{\min }^{(k)}(n)$ для всех $n>k$ и $m_{\min }^{(k)}(n)>2^{k-2} \cdot \log _{2} n$ для всех значений $n$, начиная с некоторого значения $n_{0}$.

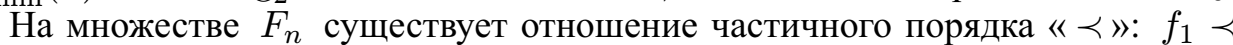
$f_{2}$ для $f_{1}, f_{2} \in F_{n}$ тогда и только тогда, когда $f_{1}\left(x_{1}, \ldots, x_{n}\right) \leqslant f_{2}\left(x_{1}, \ldots, x_{n}\right)$ для любых значений аргументов $x_{1}, \ldots, x_{n}$. Это отношение превращает $F_{n}$ в частично упорядоченное множество. Напомним ([7]), что идеалом, порожденным элементом $f$, в таком множестве называется $I(f)=\left\{f^{\prime} \in F_{n}: f \prec f^{\prime}\right\}$.

Утверждение 1. Если $f \in G_{n}^{(k)}$, то $I(f) \subset G_{n}^{(k)}$.

Доказательство. Пусть $f \in G_{n}^{(k)}$. Рассмотрим $f^{\prime} \in I(f)$, тогда $E_{f} \subset E_{f^{\prime}}$. Предположим, что у $f^{\prime}$ есть имплицента $g$, что эквивалентно включению $E_{f^{\prime}} \subset E_{g}$. Но тогда $E_{f} \subset E_{g}$, что эквивалентно равенству $f \cdot g=f$, а это противоречит условию $f \in G_{n}^{(k)}$.

Из утверждения 1 следует, что построение функции $f \in G_{n}^{(k)}$ дает возможность построения целого класса функций $\left\{f \vee f^{\prime}: f^{\prime} \in F_{n}\right\} \subset G_{n}^{(k)}$ без имплицент от $k$ переменных. Мощность этого класса тем больше, чем меньше вес функции $f$, что делает актуальным построение алгоритмов нахождения функций $f \in G_{n}^{(k)}$ как можно более малого веса.

В параграфе 1 введено понятие $k$-сюръективного семейства булевых функций и предложен способ построения комбинаторно полных матриц порядка $k$ на основе такого семейства. В параграфе 2 построено несколько $k$ сюръективных семейств и найдены верхние оценки их мощностей. В параграфе 3 рассмотрен вопрос о возможности наличия повторяющихся строк в построенной по $k$-сюръективному семейству комбинаторно полной матрице.

\section{1. Определение $k$-сюръективного семейства булевых функций и построение комбинаторно полных матриц порядка $k$}

При $k \geqslant 2$ семейство $M$ булевых функций от переменных $x_{1}, \ldots, x_{k-1}$ назовем $k$-сюръективнылм, если для любых различных векторов $\bar{\beta}_{1}, \ldots, \bar{\beta}_{k} \in$ $V_{k-1}$ и для любых $a_{1}, \ldots, a_{k} \in\{0,1\}$ найдется такая функция $f \in M$, что $f\left(\bar{\beta}_{1}\right)=a_{1}, \ldots, f\left(\bar{\beta}_{k}\right)=a_{k}$. 
Отметим, что $\left|V_{k-1}\right|=2^{k-1} \geqslant k$ при $k \geqslant 2$, поэтому всегда существует хотя бы один набор различных векторов $\bar{\beta}_{1}, \ldots, \bar{\beta}_{k} \in V_{k-1}$.

Для любого $k \geqslant 2$ существуют $k$-сюръективные семейства: очевидно, что $k$-сюръективным семейством является множество $F_{k-1}$. Другие примеры таких семейств приведены в следующем параграфе.

Укажем способ построения комбинаторно полных матриц порядка $k$. Пусть $k>1$ и $R(k) \subset F_{k-1}-$ семейство булевых функций от переменных $x_{1}, \ldots, x_{k-1}$. Рассмотрим произвольное $r \geqslant k$ и множество булевых переменных $y_{1}, \ldots, y_{r}$. Построим семейство $R(k, r)$ булевых функций от этих переменных следующим образом: для каждого набора $1 \leqslant i_{1}<i_{2}<\cdots<$ $i_{k-1} \leqslant r$ и каждой функции $f\left(x_{1}, \ldots, x_{k-1}\right) \in R(k)$ в семейство $R(k, r)$ добавим функцию $F_{i_{1}, \ldots, i_{k-1}}^{f}\left(y_{1}, \ldots, y_{r}\right)=f\left(y_{i_{1}}, \ldots, y_{i_{k-1}}\right)$. Очевидно, что $|R(k, r)|=\left(\begin{array}{c}r \\ k-1\end{array}\right) \cdot|R(k)|$.

Зададим вектором значений $\left(F_{i_{1}, \ldots, i_{k-1}}^{f}(0, \ldots, 0), \quad F_{i_{1}, \ldots, i_{k-1}}^{f}(0, \ldots, 0,1)\right.$, $\left.\mathrm{F}_{i_{1}, \ldots, i_{k-1}}^{f}(0, \ldots, 1,0), \ldots F_{i_{1}, \ldots, i_{k-1}}^{f}(1, \ldots, 1)\right)$, где аргументы (векторы длины $r$ ) перечислены в лексикографическом порядке, каждую из функций $F_{i_{1}, \ldots, i_{k-1}}^{f} \in R(k, r)$. Используя все векторы $\vec{F}_{i_{1}, \ldots, i_{k-1}}^{f}, F_{i_{1}, \ldots, i_{k-1}}^{f} \in R(k, r)$ в качестве строк, составим из них матрицу $M\langle R(k, r)\rangle$. Эта матрица имеет $2^{r}$ столбцов и $|R(k, r)|$ строк, назовем ее построенной по семейству $R(k)$.

Теорема 2. Если семейство $R(k), k>1$, есть $k$-сюръективное семейство функиий, то матрииа $M\langle R(k, r)\rangle$, построенная по семейству $R(k)$, является комбинаторно полной порядка $k$.

Доказательство. Используем следующее обозначение: для $(0,1)$-матрицы $B$ из $k$ строк и $r$ столбцов через $B_{t \times\left\{i_{1}, \ldots, i_{s}\right\}}$ обозначим подматрицу матрицы $B$, стоящую на пересечении первых $t$ строк и столбцов с номерами $i_{1}, \ldots, i_{s}$.

Лемма 1. Пусть в $(0,1)$-матрице $B$ размера $k \times r, r \geqslant k>1$, все строки различны. Тогда существует такое подмножество $\left\{i_{1}, i_{2}, \ldots, i_{k-1}\right\} \subset$ $\{1,2, \ldots, r\}$, что в матриче $B_{k \times\left\{i_{1}, \ldots, i_{k-1}\right\}}$ все строки также различны.

Доказательство. Применим индукцию по $k$. При $k=2$ утверждение леммы очевидно, поскольку означает, что в матрице, состоящей из двух различных строк, найдется такой номер столбца $i_{1}$, что на пересечении $i_{1}$-го столбца с одной строкой стоит 0 , а на пересечении с другой строкой стоит 1 .

Пусть теперь лемма верна при всех $k^{\prime}<k$, докажем ее для $k^{\prime}=k$. Так как в матрице $B$ все строки различны, то при отбрасывании из $B$ последней строки это свойство сохранится, т. е. в матрице $B_{(k-1) \times\{1,2, \ldots, r\}}$ также все строки различны. По предположению индукции существует такое подмножество $\left\{i_{1}, i_{2}, \ldots, i_{k-2}\right\} \subset\{1,2, \ldots, r\}$, что в матрице $B_{(k-1) \times\left\{i_{1}, \ldots, i_{k-2}\right\}}$ все строки различны. 
Если последняя строка в матрице $B_{k \times\left\{i_{1}, \ldots, i_{k-2}\right\}}$ не совпадает с другими строками этой матрицы, то в матрице $B_{k \times\left\{i_{1}, \ldots, i_{k-2}\right\}}$ все строки различны и, следовательно, при любом $j \in\{1, \ldots, r\} \backslash\left\{i_{1}, \ldots, i_{k-2}\right\}$ в матрице $B_{k \times\left\{i_{1}, \ldots, i_{k-2}, j\right\}}$ все строки различны, т. е. $B_{k \times\left\{i_{1}, \ldots, i_{k-2}, j\right\}}$ является искомой подматрицей.

Обозначим строки матрицы $B_{k \times\left\{i_{1}, \ldots, i_{k-2}\right\}}$ через $\vec{T}_{1}, \ldots, \vec{T}_{k}$. Если, в отличие от предыдущего случая, последняя строка $\vec{T}_{k}$ совпадает с некоторой строкой $\vec{T}_{u}, u \in\{1, \ldots, k-1\}$, то в силу того, что в матрице $B_{(k-1) \times\left\{i_{1}, \ldots, i_{k-2}\right\}}$ все строки различны, $\vec{T}_{u}$ не совпадает с $\vec{T}_{v}$ при $u \neq v, v \in\{1, \ldots, k-1\}$. Найдем среди номеров столбцов $\{1, \ldots, r\} \backslash\left\{i_{1}, \ldots, i_{k-2}\right\}$ матрицы $B$ такой номер $j$, что строки с номерами $k$ и $u$ на пересечении с $j$-м столбцом содержат различные значения (такой номер найдется, поскольку в координатах с номерами из $\left\{i_{1}, \ldots, i_{k-2}\right\}$ указанные строки совпадают, а матрица $B$ не содержит одинаковых строк). Тогда в матрице $B_{k \times\left\{i_{1}, \ldots, i_{k-2, j}\right\}}$ нет одинаковых строк, т. е. она является искомой подматрицей.

Вернемся к доказательству теоремы. Проиндексируем столбцы матрицы $M\langle R(k, r)\rangle$ битовыми векторами длины $r$ в лексикографическом порядке. Тогда по определению матрицы $M\langle R(k, r)\rangle$ на пересечении столбца с номером $\bar{\gamma} \in V_{r}$ и строки, равной $\vec{F}_{i_{1}, \ldots, i_{k-1}}^{f}=\left(F_{i_{1}, \ldots, i_{k-1}}^{f}(0, \ldots, 0)\right.$, $\left.F_{i_{1}, \ldots, i_{k-1}}^{f}(0, \ldots, 0,1), \ldots, F_{i_{1}, \ldots, i_{k-1}}^{f}(1, \ldots, 1)\right), \quad$ стоит $\quad$ элемент, $\quad$ равный $F_{i_{1}, \ldots, i_{k-1}}^{f}(\bar{\gamma})$. Проверим, выполняются ли для матрицы $M\langle R(k, r)\rangle$ условия комбинаторной полноты порядка $k$. Возьмем $k$ различных столбцов матрицы $M\langle R(k, r)\rangle$, индексированных векторами $\bar{\gamma}_{1}, \ldots, \bar{\gamma}_{k} \in V_{r}$. Столбцы различны, значит, и векторы различны. Применим к матрице $B=\left(\bar{\gamma}_{1} \cdots \bar{\gamma}_{k}\right)^{\mathrm{T}}$ лемму и получим, что существует такое подмножество $\left\{i_{1}, i_{2}, \ldots, i_{k-1}\right\} \subset\{1,2, \ldots, r\}$, что в матрице $B_{k \times\left\{i_{1}, \ldots, i_{k-1}\right\}}$ все строки также различны. Без ограничения общности считаем, что числа в подмножестве упорядочены по возрастанию, т.е. $1 \leqslant i_{1}<i_{2}<\cdots<i_{k-1} \leqslant r$. Обозначим $B_{k \times\left\{i_{1}, \ldots, i_{k-1}\right\}}=\left(\bar{\beta}_{1} \cdots \bar{\beta}_{k}\right)^{\mathrm{T}}$. Теперь рассмотрим произвольный набор $a_{1}, \ldots, a_{k} \in\{0,1\}$. Поскольку семейство $R(k)$ является $k$-сюръективным семейством и набор $\bar{\beta}_{1}, \ldots, \bar{\beta}_{k}$ состоит из различных векторов, по определению найдется такая функция $f \in R(k)$, что $f\left(\bar{\beta}_{1}\right)=a_{1}$, $\ldots, f\left(\bar{\beta}_{k}\right)=a_{k}$. Рассмотрим в семействе $R(k, r)$ функцию $F_{i_{1}, \ldots, i_{k-1}}^{f}$, которая согласно своему определению задается следующим образом: $F_{i_{1}, \ldots, i_{k-1}}^{f}\left(\alpha_{1}, \ldots, \alpha_{r}\right)=f\left(\alpha_{i_{1}}, \ldots, \alpha_{i_{k-1}}\right)$ для любого $\left(\alpha_{1}, \ldots, \alpha_{r}\right) \in V_{r}$. Отсюда следует, что $F_{i_{1}, \ldots, i_{k-1}}^{f}\left(\bar{\gamma}_{i}\right)=f\left(\bar{\beta}_{i}\right)=a_{i}, \quad i=\overline{1, k}$. 
Таким образом, в строке матрицы $M\langle R(k, r)\rangle$, равной вектору значений функции $F_{i_{1}, \ldots, i_{k-1}}^{f}$, на пересечении со столбцами, проиндексированными векторами $\bar{\gamma}_{1}, \ldots, \bar{\gamma}_{k} \in V_{r}$, стоят значения $a_{1}, \ldots, a_{k}$ соответственно. Поскольку строка $\left(a_{1}, \ldots, a_{k}\right)$ может принимать все $2^{k}$ возможных значений, матрица $M\langle R(k, r)\rangle$ является комбинаторно полной порядка $k$.

Из проведенных выше рассуждений получаем следующий метод построения комбинаторно полных матриц порядка $k$ с $n$ строками. Выберем значение $r=\max \left\{\left\lceil\log _{2} n\right\rceil, k\right\}$, где $\lceil x\rceil-$ наименьшее целое, не меньшее $x$. Далее возьмем переполненное семейство булевых функций $R(k)$ и матрицу $M\langle R(k, r)\rangle$, построенную по этому семейству. По теореме 2 эта матрица комбинаторно полная порядка $k$ и число столбцов в этой матрице равно $2^{r} \geqslant n$. Если $r>\log _{2} n$, то, отбросив произвольные $2^{r}-n$ столбцов, получим комбинаторно полную матрицу $A$ порядка $k$ с $n$ столбцами. Число строк в матрице $A$ равно $|R(k, r)|=\left(\begin{array}{c}r \\ k-1\end{array}\right) \cdot|R(k)|$.

Сделаем несколько замечаний. В матрицах $A$ и $M\langle R(k, r)\rangle$ могут оказаться совпадающие строки; очевидно, что повторы можно удалить без ущерба для комбинаторной полноты матрицы. Критерий того, что матрица $M\langle R(k, r)\rangle$ содержит повторяющиеся строки, приведен в параграфе 3. Необходимо заметить, что, вообще говоря, даже если матрица $M\langle R(k, r)\rangle$ не имеет повторов, то после отбрасывания некоторых столбцов в матрице $A$ повторы могут появиться; поэтому даже в случаях, когда удается точно вычислить количество несовпадающих строк в матрице $M\langle R(k, r)\rangle$, это количество может служить только оценкой сверху для аналогичного параметра матрицы $A$. Кроме того, вполне естественно искать $k$-сюръективное семейство как можно меньшей мощности, чтобы уменьшить размер матрицы $M\langle R(k, r)\rangle$.

\section{2. Примеры $k$-сюръективных семейств булевых функций}

Как видно из предыдущего параграфа, используемое при построении комбинаторно полной матрицы $k$-сюръективное семейство должно быть как можно меньшей мощности. Поэтому сначала приведем некоторые оценки минимально возможного значения мощности $k$-сюръективного семейства. Нижняя оценка получается довольно просто.

Утверждение 2. Любое $k$-сюръективное семейство имеет мощность не менее $2^{k}$.

Доказательство. В определении $k$-сюръективного семейства $R(k)$ зафиксируем набор различных векторов $\bar{\beta}_{1}, \ldots, \bar{\beta}_{k} \in V_{k-1}$ и рассмотрим $2^{k}$ различных наборов $a_{1}, \ldots, a_{k}$. Каждому варианту $\left(a_{1}, \ldots, a_{k}\right)$ соответствует такая функция $f$ из $R(k)$, что $f\left(\bar{\beta}_{1}\right)=a_{1}, \ldots, f\left(\bar{\beta}_{k}\right)=a_{k}$; очевидно, разным наборам соответствуют разные функции. Следовательно, в семействе $R(k)$ содержится не менее $2^{k}$ функций. 
Следующее утверждение показывает, что минимальное значение мощности $k$-сюръективного семейства достаточно близко к доказанной нижней оценке.

Утверждение 3. Существует $k$-сюръективное семейство мощности не более $2^{k} \cdot\left(k^{2}-k+2\right)$.

Доказательство. Рассмотрим булеву матрицу $G(k)$, строки которой проиндексированы функциями из $F_{k-1}$, столбцы - упорядоченными парами $\left(\left(\bar{\beta}_{1}, \ldots, \bar{\beta}_{k}\right),\left(a_{1}, \ldots, a_{k}\right)\right)$, где $\bar{\beta}_{1}, \ldots, \bar{\beta}_{k} \in V_{k-1}$ различны и $a_{1}, \ldots, a_{k} \in\{0,1\}$. Тогда число строк матрицы $G(k)$ равно $2^{2^{k-1}}$, число столбцов равно $\left(\begin{array}{c}2^{k-1} \\ k\end{array}\right) \cdot k ! \cdot 2^{k}$. На пересечении строки, соответствующей функции $f\left(x_{1}, \ldots, x_{k-1}\right)$, и столбца, индексированного парой $\left(\left(\bar{\beta}_{1}, \ldots, \bar{\beta}_{k}\right),\left(a_{1}, \ldots, a_{k}\right)\right)$, поставим единицу тогда и только тогда, когда $f\left(\bar{\beta}_{1}\right)=a_{1}, \ldots, f\left(\bar{\beta}_{k}\right)=a_{k}$. Тогда каждый столбец содержит $2^{2^{k-1}-k}$ единиц, поскольку в столбце стоят единицы в строках, соответствующих функциям, у которых $k$ значений из всех $2^{k-1}$ возможных значений зафиксированы.

Напомним ([8]), что в произвольной булевой матрице $B$ строка и столбец называются пересекающимися, если на месте их пересечения стоит 1; также говорят, что строка покрывает столбцы, с которыми пересекается. Кроме того, покрытием булевой матрицы $B$ называется такое подмножество $M$ ее строк, что для любого столбца матрицы $B$ найдется строка из $M$, пересекающаяся с этим столбцом. Пусть матрица $B$ имеет $p$ строк, $s$ столбцов и в каждом столбце не менее $p \cdot \gamma$ единиц, $0<\gamma \leqslant 1$. В [4] доказана теорема о том, что если $s \cdot \gamma \geqslant 1$, то «жадный» алгоритм построения покрытия, т. е. алгоритм, добавляющий на каждом шаге в строящееся покрытие строку, пересекающуюся с наибольшим количеством еще не покрытых столбцов, дает покрытие, имеющее мощность не более $\left|\gamma^{-1} \cdot \ln (\gamma \cdot s)\right|+\gamma^{-1}$ (здесь $\lceil x\rceil=\min _{\nu \in \mathbb{Z}}\{\nu \geqslant x\}$ ).

Применим эту теорему к $G(k)$, учитывая, что в этом случае $p=2^{k-1}$, $s=\left(\begin{array}{c}2^{k-1} \\ k\end{array}\right) \cdot k ! \cdot 2^{k}$ и в каждом столбце ровно $2^{2^{k-1}}=p \cdot \gamma$ единиц, где $\gamma=2^{-k}$. Тогда $s \cdot \gamma=\left(\begin{array}{c}2^{k-1} \\ k\end{array}\right) \cdot k ! \geqslant 1$ и «жадный» алгоритм дает покрытие матрицы $G(k)$ не более чем $\left\lceil 2^{k} \cdot \ln \left(\left(\begin{array}{c}2^{k-1} \\ k\end{array}\right) \cdot k !\right)\right\rceil+2^{k}$ строками.

Теперь покажем, что набор строк $M$ матрицы $G(k)$ является ее покрытием тогда и только тогда, когда множество булевых функций, соответствующих строкам из $M$, является $k$-сюръективным семейством. Действительно, $M$ - покрытие тогда и только тогда, когда для любого столбца матрицы $G(k)$ (т.е. для любой упорядоченной пары $\left(\left(\bar{\beta}_{1}, \ldots, \bar{\beta}_{k}\right),\left(a_{1}, \ldots, a_{k}\right)\right)$, где $\bar{\beta}_{1}, \ldots, \bar{\beta}_{k} \in V_{k-1}$ различны и $\left.a_{1}, \ldots, a_{k} \in\{0,1\}\right)$ найдется такая строка в $M$ (т. е. найдется такая функция $\left.f \in F_{k-1}\right)$, что на пересечении этой строки и столбца, соответствующего паре $\left(\left(\bar{\beta}_{1}, \ldots, \bar{\beta}_{k}\right),\left(a_{1}, \ldots, a_{k}\right)\right)$, стоит 1 (что по определению означает $\left.f\left(\bar{\beta}_{1}\right)=a_{1}, \ldots, f\left(\bar{\beta}_{k}\right)=a_{k}\right)$. 
Значит, «жадный» алгоритм построит $k$-сюръективное семейство мощности не более $\left\lceil 2^{k} \cdot \ln \left(\left(\begin{array}{c}2^{k-1} \\ k\end{array}\right) \cdot k !\right)\right\rceil+2^{k}$. Далее, используя неравенства $\left(\begin{array}{l}n \\ k\end{array}\right)<\frac{n^{k}}{k !}$ при $k \geqslant 2,\lceil x\rceil \leqslant x+1$ и $\ln 2<1$, получим:

$$
\begin{aligned}
& {\left[2^{k} \cdot \ln \left(\left(\begin{array}{c}
2^{k-1} \\
k
\end{array}\right) \cdot k !\right)\right]+2^{k}<\left[2^{k} \cdot \ln \left(\left(2^{k-1}\right)^{k}\right)\right]+2^{k} \leqslant} \\
& \leqslant 2^{k} \cdot \ln \left(\left(2^{k-1}\right)^{k}\right)+2^{k}+1=2^{k} \cdot\left(\left(k^{2}-k\right) \cdot \ln 2+1\right)+1< \\
& <2^{k} \cdot\left(k^{2}-k+1\right)+1 .
\end{aligned}
$$

Так как $\left\lceil 2^{k} \cdot \ln \left(\left(\begin{array}{c}2^{k-1} \\ k\end{array}\right) \cdot k !\right)\right\rceil+2^{k}-$ целое число, то $\left\lceil 2^{k} \cdot \ln \left(\left(\begin{array}{c}2^{k-1} \\ k\end{array}\right) \cdot k !\right)\right\rceil+2^{k} \leqslant$ $\leqslant 2^{k} \cdot\left(k^{2}-k+1\right)$.

Доказательство этого утверждения дает метод построения $k$-сюръективного семейства булевых функций путем применения «жадного» алгоритма к построению покрытия булевой матрицы размера $\left(\begin{array}{c}2^{k-1} \\ k\end{array}\right) \cdot k ! \cdot 2^{k} \times 2^{2^{k-1}}$. Очевидно, что такие большие размеры матрицы делают невозможным практическое применение алгоритма уже при $k \geqslant 7$. Согласно результатам предыдущего параграфа, нахождение $k$-сюръективного семейства $R(k)$ минимальной мощности позволит построить комбинаторно полную матрицу $M\langle R(k, r)\rangle$ порядка $k$ с $n=2^{r}$ столбцами и числом строк, не превосходящим

$$
\left(\begin{array}{l}
\log _{2} n \\
k-1
\end{array}\right) \cdot 2^{k} \cdot\left(k^{2}-k+1\right) .
$$

Далее приведем несколько примеров $k$-сюръективных семейств, лежащих в $F_{k-1}$, и оценки их мощностей. Отметим, что найденные семейства далеки от минимальных, за исключением последнего семейства.

1. Семейство $M(k), k \geqslant 2$. Как уже указывалось выше, очевидно, что множество $M(k)=F_{k-1}$ является $k$-сюръективным семейством. Мощность такого семейства составляет $2^{2^{k-1}}$.

2. Семейство $H(k), k \geqslant 2$. Определим булеву функцию $f_{\bar{\beta}_{1}, \ldots, \bar{\beta}_{k}}^{a_{1}, \ldots, a_{k}}\left(x_{1}, \ldots, x_{k-1}\right)$ следующим образом:

$$
f_{\bar{\beta}_{1}, \ldots, \bar{\beta}_{k}}^{a_{1}, \ldots, a_{k}}\left(\bar{\beta}_{1}\right)=a_{1}, \ldots, f_{\bar{\beta}_{1}, \ldots, \bar{\beta}_{k}}^{a_{1}, \ldots, a_{k}}\left(\bar{\beta}_{k}\right)=a_{k}
$$

для любых $a_{1}, \ldots, a_{k} \in\{0,1\}$ и каждого зафиксированного упорядоченного набора различных $\bar{\beta}_{1}, \ldots, \bar{\beta}_{k} \in V_{k-1}$ (корректность такого определения следует из того, что векторы $\bar{\beta}_{1}, \ldots, \bar{\beta}_{k}$ различны) и $f_{\bar{\beta}_{1}, \ldots, \bar{\beta}_{k}}^{a_{1}, \ldots, a_{k}}(\bar{\beta})=0$ для векторов $\bar{\beta} \in V_{k-1}$, отличных от $\bar{\beta}_{1}, \ldots, \bar{\beta}_{k}$.

Обозначим через $H(k)$ множество всех функций $f_{\bar{\beta}_{1}, \ldots, \bar{\beta}_{k}}^{a_{1}, \ldots, a_{k}}\left(x_{1}, \ldots, x_{k-1}\right)$. Из самой процедуры построения семейства $H(k)$ очевидно следует, что оно является $k$-сюръективным. 
Две функции $f_{\bar{\beta}_{1}, \ldots, \bar{\beta}_{k}}^{a_{1}, \ldots, a_{k}}, f_{\bar{\beta}_{1}^{\prime}, \ldots, \bar{\beta}_{k}^{\prime}}^{a_{1}^{\prime}, \ldots, a^{\prime}}$ могут совпадать; подсчитаем число различных функций. Очевидно, что две функции из $H(k)$ совпадают тогда и только тогда, когда совпадают множества векторов, на которых функции принимают значение 1 . Веса функций из $H(k)$ принимают значения от 0 до $k$, и каждая функция, вес которой принимает значение из указанного интервала, является одной из функций $f_{\bar{\beta}_{1}, \ldots, \bar{\beta}_{k}}^{a_{1}, \ldots, a_{k}}$. Значит,

$$
|H(k)|=\sum_{s=0}^{k}\left(\begin{array}{c}
2^{k-1} \\
s
\end{array}\right) \text {. }
$$

Используя простую оценку $\left(\begin{array}{l}n \\ k\end{array}\right) \leqslant \frac{n^{k}}{k !}$, получаем

$$
|H(k)| \leqslant\left(\begin{array}{c}
2^{k-1} \\
k
\end{array}\right) \cdot(k+1) \leqslant\left(2^{k-1}\right)^{k}=2^{k^{2}-k},
$$

т. е. $|H(k)| \leqslant 2^{k^{2}-k}$.

Очевидно, что $H(2)=M(2)=F_{1}$. Далее, $|H(3)|=15$, т. е. из всех булевых функций от двух переменных семейство $H(3)$ не содержит только одну функцию (функцию, тождественно равную 1). При $k \geqslant 4$ семейство $H(k)$ в определенном смысле избыточно: оно содержит собственное подмножество, также являющееся $k$-сюръективным семейством. Это семейство описано далее.

3. Семейство $E(k), k \geqslant 4$. Рассмотрим семейство $E(k) \subset H(k)$, которое состоит только из функций $f_{\bar{\beta}_{1}, \ldots, \bar{\beta}_{k}}^{1, \ldots, 1}\left(x_{1}, \ldots, x_{k-1}\right)$, т. е. из всех булевых функций веса $k$ от $k-1$ переменных. Очевидно, что $|E(k)|=\left(\begin{array}{c}2^{k-1} \\ k\end{array}\right)$. Докажем, что при $k \geqslant 4$ семейство $E(k)$ является $k$-сюръективным. Рассмотрим произвольный упорядоченный набор различных векторов $\bar{\beta}_{1}, \ldots, \bar{\beta}_{k} \in V_{k-1}$ и любые $a_{1}, \ldots, a_{k} \in\{0,1\}$. Пусть среди $a_{1}, \ldots, a_{k}$ те $a_{i}$, которые равны 1 , имеют номера $j_{1}, \ldots, j_{s}$, а остальные $a_{i}$ равны 0 . Рассмотрим функцию $f_{\bar{\beta}_{j_{1}}, \ldots, \bar{\beta}_{j_{s}}, \bar{\gamma}_{s+1}, \ldots, \bar{\gamma}_{k}}^{1, \ldots,{ }_{1}}\left(x_{1}, \ldots, x_{k-1}\right) \in E(k)$, где $\bar{\gamma}_{s+1}, \ldots, \bar{\gamma}_{k} \in V_{k-1}$ - произвольные различные векторы, отличные от векторов $\bar{\beta}_{1}, \ldots, \bar{\beta}_{k}$. Такие $\bar{\gamma}_{s+1}, \ldots, \bar{\gamma}_{k}$ найдутся, так как $\left|V_{k-1}\right|-k=2^{k-1}-k$ и, поскольку $s \geqslant 0 \geqslant 2 k-2^{k-1}$ при $k \geqslant 4$, имеем $2^{k-1}-k \geqslant k-s$. Тогда

$$
f_{\bar{\beta}_{j_{1}}, \ldots, \bar{\beta}_{j_{s}}, \bar{\gamma}_{s+1}, \ldots, \bar{\gamma}_{k}}^{1, \ldots, 1}\left(\bar{\beta}_{i}\right)=\left\{\begin{array}{lll}
1=a_{i}, & \text { если } & i \in\left\{j_{1}, \ldots, j_{s}\right\}, \\
0=a_{i}, & \text { если } & i \in\{1, \ldots, k\} \backslash\left\{j_{1}, \ldots, j_{s}\right\} .
\end{array}\right.
$$

Значит, семейство $E(k)$ является $k$-сюръективным. 
4. Семейство $S(k), k=2 s, s \geqslant 1$. Рассмотрим $k$-сюръективное семейство, идею построения которого предложил А.Б. Шишков. Это семейство имеет не комбинаторный, а алгебраический способ задания; используемые определения можно найти в [5]. Пусть $Q=G F\left(2^{k-1}\right)$ и $P=G F(2)$. Через $\operatorname{tr}(\alpha)=$ $\alpha+\alpha^{2}+\cdots+\alpha^{2^{k-2}}$ обозначим след элемента $\alpha \in Q$ в поле $P$. Выберем в поле $Q$ некоторый базис над полем $P$; очевидно, в этом базисе $k-1$ элементов. Тогда каждый элемент $\alpha \in Q$ можно рассматривать как двоичный вектор размерности $k-1$, компоненты которого являются координатами $\alpha$ в выбранном базисе, и любое отображение из $Q$ в $P$ в указанном смысле есть булева функция от $k-1$ переменных.

Следом полинома $p(x) \in Q[x]$ называется отображение $\operatorname{tr}(p(x)): Q \rightarrow$ $P$, значение которого на элементе $\alpha \in Q$ равно $\operatorname{tr}(p(\alpha))$. Отметим, что у двух полиномов $p_{1}(x)$ и $p_{2}(x)$ следы могут совпадать. Из четности значения $k$ следует, что если $p(\alpha) \in P$ для полинома $p(x) \in Q[x]$ и некоторого элемента $\alpha \in Q, \operatorname{to~} \operatorname{tr}(p(\alpha))=p(\alpha)$. Действительно,

$$
\operatorname{tr}(p(\alpha))=0+0^{2}+\cdots+0^{2^{k-2}}=0, \quad \text { если } \quad p(\alpha)=0,
$$

И

$$
\operatorname{tr}(p(\alpha))=1+1^{2}+\cdots+1^{2^{k-2}}=\underbrace{1+\cdots+1}_{k-1}=1, \quad \text { если } \quad p(\alpha)=1 .
$$

Далее рассмотрим множество $Q^{(k)}[x]$ всех многочленов степени $k$ над полем $Q$ и множество $\operatorname{tr}\left(Q^{(k)}[x]\right)$, состоящее из всех таких отображений $\xi: Q \rightarrow P$, которые совпадают с $\operatorname{tr}(p(x))$ для некоторого многочлена $p(x) \in Q^{(k)}[x]$. Проще говоря, $\operatorname{tr}\left(Q^{(k)}[x]\right)$ есть множество всех различных следов многочленов над полем $Q$ степени $k$. Как указано выше, каждое отображение из $\operatorname{tr}\left(Q^{(k)}[x]\right)$ есть булева функция от $k-1$ переменных. Докажем, что $\operatorname{tr}\left(Q^{(k)}[x]\right)$ является $k$-сюръективным семейством.

В указанном выше смысле каждый вектор из $V_{k-1}$ рассматриваем как элемент поля $Q$. Набору различных $\bar{\beta}_{1}, \ldots, \bar{\beta}_{k} \in V_{k-1}$ и любых $a_{1}, \ldots, a_{k} \in$ $\{0,1\}$ поставим в соответствие многочлен $p(x) \in Q[x]$, задаваемый интерполяционной формулой Лагранжа. Тогда $p\left(\bar{\beta}_{1}\right)=a_{1}, \ldots, p\left(\bar{\beta}_{k}\right)=a_{k}$ и $\operatorname{deg} p(x)=k$. Значит, $p(x) \in Q^{(k)}[x]$, и поэтому $\operatorname{tr}(p(x)) \in \operatorname{tr}\left(Q^{(x)}[x]\right)$. Как показано выше, $\operatorname{tr}(p(x))$ есть булева функция от $k-1$ переменных и $\operatorname{tr}\left(p\left(\bar{\beta}_{i}\right)\right)=a_{i}, i \in\{1, \ldots, k\}$, поскольку $p\left(\bar{\beta}_{i}\right) \in P, i \in\{1, \ldots, k\}$. Следовательно, семейство $\operatorname{tr}\left(Q^{(k)}[x]\right)$ является $k$-сюръективным. 
Теперь оценим мощность семейства $\operatorname{tr}\left(Q^{(x)}[x]\right)$.

Утверждение 4. Если $k \geqslant 2$ четно, то $\left|\operatorname{tr}\left(Q^{(x)}[x]\right)\right| \leqslant 2^{\frac{1}{2}\left(k^{2}-k\right)}$.

Доказательство. Обозначим через $Q^{\leqslant k}[x]$ множество полиномов над $Q$ степени не более $k$, через $F(Q)$ - множество всех отображений из $Q$ в $Q$. Операция суммирования элементов поля $Q$ задает аналогичные операции суммирования многочленов и отображений и позволяет рассмотреть группы $(Q[x],+), \quad\left(Q^{\leqslant k}[x],+\right)$ и $(F(Q),+)$. Взятие следа многочлена задает гомоморфизм $\operatorname{tr}$ из $(Q[x],+)$ в $(F(Q),+)$. Сужение этого гомоморфизма на подгруппу $\left(Q^{\leqslant k}[x],+\right)$ дает гомоморфизм $\operatorname{tr}^{(k)}$ из $\left(Q^{\leqslant k}[x],+\right)$ на $\operatorname{tr}\left(Q^{\leqslant k}[x]\right)$. Поэтому

$$
\left|\left(Q^{\leqslant k}[x],+\right) / \operatorname{Ker}^{(k)}\right|=\left|\operatorname{tr}\left(Q^{\leqslant k}[x]\right)\right| .
$$

Отметим, что $Q^{(k)}[x] \subset Q^{\leqslant k}[x]$, следовательно,

$$
\operatorname{tr}\left(Q^{(k)}[x]\right) \subset \operatorname{tr}\left(Q^{\leqslant k}[x]\right) \leqslant\left|\operatorname{tr}\left(Q^{\leqslant k}[x]\right)\right| .
$$

Ядро гомоморфизма $\operatorname{tr}$ известно ([5]): $\operatorname{Ker} \operatorname{tr}=\left\{q(x)+q^{2}(x): q(x) \in Q[x]\right\}$. Очевидно, что $\operatorname{Kertr}^{(k)}=\operatorname{Ker} \operatorname{tr} \cap Q^{\leqslant k}[x]$. Значит,

$$
\text { Kertr } \operatorname{tr}^{(k)}=\left\{q(x)+q^{2}(x): q(x) \in Q[x] \text { и } \operatorname{deg}\left(q(x)+q^{2}(x)\right) \leqslant k\right\} .
$$

Следовательно, в $\operatorname{Ker}_{\operatorname{tr}}^{(k)}$ входят те и только те полиномы, которые имеют вид $q(x)+q^{2}(x)$, где $q(x) \in Q^{\leqslant k / 2}[x]$. Значит,

$$
\mid \text { Ker } \operatorname{tr}^{(k)} \mid=\left(2^{k-1}\right)^{k / 2+1} .
$$

Очевидно, что $\left|Q^{\leqslant k}[x]\right|=\left(2^{k-1}\right)^{k+1}$, поэтому

$$
\left|\operatorname{tr}\left(Q^{(k)}[x]\right)\right| \leqslant\left|\operatorname{tr}\left(Q^{\leqslant k}[x]\right)\right|=\frac{\left(2^{k-1}\right)^{k+1}}{\left(2^{k-1}\right)^{k / 2+1}}=2^{\left(k^{2}-k\right) / 2} .
$$

5. Семейство $T(k), k=2,3$. Рассмотрим семейство функций $T(k)$, состоящее из функций, тождественно равных константе, и всех линейных и аффинных функций от переменных $x_{1}, \ldots, x_{k-1}$, т. е. из функций вида

$$
f\left(x_{1}, \ldots, x_{k-1}\right)=c_{1} \cdot x_{1} \oplus \cdots \oplus c_{k-1} \cdot x_{k-1} \oplus b,
$$

где $c_{1}, \ldots, c_{k-1}, b \in\{0,1\}$. 
Утверждение 5. При $k \in\{2,3\}$ семейство $T(k)$ является $k$-сюръективным семейством.

Доказательство. Рассмотрим случай $k=2$. По определению $T(2)$ состоит из 4 различных функций от одной переменной, т. е. $T(2)$ содержит все булевы функции от одной переменной и, очевидно, является 2-сюръективным семейством.

Пусть теперь $k=3$ и $T(3)=\left\{c_{1} \cdot x_{1} \oplus c_{2} \cdot x_{2} \oplus b: c_{1}, c_{2}, b \in\{0,1\}\right\}$. По определению $T(3)$ является 3 -сюръективным семейством тогда и только тогда, когда для любых различных $\bar{v}=\left(v_{1}, v_{2}\right), \bar{w}=\left(w_{1}, w_{2}\right), \bar{z}=\left(z_{1}, z_{2}\right)$ из $V_{2}$ и для любых $a_{1}, a_{2}, a_{3} \in\{0,1\}$ в $T(3)$ найдется такая функция $f$, что $f(\bar{v})=a_{1}, f(\bar{w})=a_{2}, f(\bar{z})=a_{3}$. Это можно переписать в матричном виде

$$
\left(c_{1}, c_{2}, b\right) \cdot\left(\begin{array}{ccc}
v_{1} & w_{1} & z_{1} \\
v_{2} & w_{2} & z_{2} \\
1 & 1 & 1
\end{array}\right)=\left(a_{1}, a_{2}, a_{3}\right) .
$$

Таким образом, 3-сюръективность семейства $T(3)$ эквивалентна тому, что для любой булевой матрицы

$$
\left(\begin{array}{ccc}
v_{1} & w_{1} & z_{1} \\
v_{2} & w_{2} & z_{2} \\
1 & 1 & 1
\end{array}\right)
$$

все столбцы которой различны, система линейных уравнений (1) относительно неизвестных $c_{1}, c_{2}, \quad b$ должна иметь решение. Докажем это, показав, что указанная матрица всегда обратима. Применяем эквивалентные преобразования

$$
\left(\begin{array}{ccc}
v_{1} & w_{1} & z_{1} \\
v_{2} & w_{2} & z_{2} \\
1 & 1 & 1
\end{array}\right) \approx\left(\begin{array}{ccc}
1 & 1 & 1 \\
v_{1} & w_{1} & z_{1} \\
v_{2} & w_{2} & z_{2}
\end{array}\right) \approx\left(\begin{array}{ccc}
1 & 1 & 1 \\
0 & w_{1} \oplus v_{1} & z_{1} \oplus v_{1} \\
0 & w_{2} \oplus v_{2} & z_{2} \oplus v_{2}
\end{array}\right)
$$

Обозначим последнюю матрицу через $A$. Так как пары $\left(v_{1}, v_{2}\right)$ и $\left(w_{1}, w_{2}\right)$ различны, то либо $w_{1} \oplus v_{1}=1$, либо $w_{2} \oplus v_{1}=1$, либо $w_{2} \oplus v_{2}=1$. Пусть далее $w_{1} \oplus v_{1}=1$ (во втором случае доказательство аналогично с точностью до перенумерации). Если $w_{2} \oplus v_{2}=0$, то $w_{2}=v_{2}$ и тогда $z_{2} \neq w_{2}=v_{2}$ (в противном случае пара $\left(z_{1}, z_{2}\right)$ совпадет либо с $\left(v_{1}, v_{2}\right)$, либо с $\left.\left(w_{1}, w_{2}\right)\right)$. Значит, $z_{2} \oplus v_{2}=1$ и

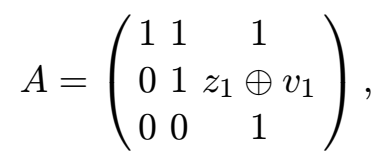

т. е. матрица $A$ обратима. 
Если $w_{2} \oplus v_{2}=1$, то

$$
A=\left(\begin{array}{ccc}
1 & 1 & 1 \\
0 & 1 & z_{1} \oplus v_{1} \\
0 & 1 & z_{2} \oplus v_{2}
\end{array}\right) \approx\left(\begin{array}{ccc}
1 & 1 & 1 \\
0 & 1 & z_{1} \oplus v_{1} \\
0 & 0 & z_{1} \oplus v_{1} \oplus z_{2} \oplus v_{2}
\end{array}\right) .
$$

Если $z_{1}=v_{1}$, то $z_{2} \neq v_{2}$ и $z_{1} \oplus v_{1} \oplus z_{2} \oplus v_{2}=1$. Если $z_{1} \neq v_{1}$, то так как $w_{1} \oplus v_{1}=1$, имеем $z_{1}=w_{1}$. Значит, $z_{2} \neq w_{2}$, и так как $w_{2} \oplus v_{2}=1$, то $z_{2}=v_{2}$. Отсюда получаем, что и в этом случае $z_{1} \oplus v_{1} \oplus z_{2} \oplus v_{2}=1$. Таким образом,

$$
f A \approx\left(\begin{array}{ccc}
1 & 1 & 1 \\
0 & 1 & z_{1} \oplus v_{1} \\
0 & 0 & 1
\end{array}\right)
$$

и поэтому $A$ обратима.

Следовательно, система (1) всегда имеет решение, а значит, семейство функций $T(3)$ является 3 -сюръективным семейством.

Стоит отметить, что семейство $T(k)$ при $k \geqslant 4$ уже не является $k$-сюръективным семейством, поскольку для этого система линейных уравнений

$$
\left(c_{1}, \ldots, c_{k-1}, b\right) \cdot\left(\begin{array}{cccc}
\beta_{1}^{(1)} & \beta_{2}^{(2)} & \ldots & \beta_{k}^{(1)} \\
\beta_{1}^{(2)} & \beta_{2}^{(2)} & \ldots & \beta_{k}^{(2)} \\
& & \ldots & \\
\beta_{1}^{(k-1)} & \beta_{2}^{(k-1)} & \ldots & \beta_{k}^{(k-1)} \\
1 & 1 & \ldots & 1
\end{array}\right)=\left(a_{1}, \ldots, a_{k}\right)
$$

должна иметь решение при любой матрице, состоящей из различных столбцов, и любой правой части. Рассмотрим матрицу

$$
\left(\begin{array}{cccc|c}
1 & 0 & 0 & 1 & \\
0 & 1 & 0 & 1 & O_{3 \times(k-4)} \\
0 & 0 & 1 & 1 & \\
\hline O_{(k-4) \times 4} & E_{(k-4) \times(k-4)} \\
\hline 1 & 1 & 1 & 1 & J_{1 \times(k-4)}
\end{array}\right)
$$

где $O_{t \times s}, J_{t \times s}, E_{t \times s}-$ матрица из нулей, матрица из единиц и единичная матрица, соответственно, с $t$ строками и $s$ столбцами. Очевидно, что в матрице (3) все столбцы различны. Пусть, далее, $\left(a_{1}, \ldots, a_{k}\right)=(1,0,0, \ldots, 0)$. Подставляя эти значения в (2), получаем, что $c_{1} \oplus b=1, c_{2} \oplus b=0, c_{3} \oplus b=0$ и $c_{1} \oplus c_{2} \oplus c_{3} \oplus b=0$, что приводит к противоречию. Следовательно, в данном случае система (2) не имеет решения, значит, соответствующее семейство не является $k$-сюръективным. 


\section{3. Совпадающие строки в матрице $M\langle R(k, r)\rangle$}

Чтобы избежать неоднозначного толкования, определим, что в булевой матрице любые ее две строки назовем несовпадающими, если они не равны как двоичные векторы, и совпадающими в противном случае.

В матрице $M\langle R(k, r)\rangle$, построенной по $k$-сюръективному семейству $R(k)$, могут содержаться совпадающие строки. Следующее утверждение полностью характеризует такой случай.

Теорема 3. Пусть матрица $M\langle R(k, r)\rangle$ построена по $k$-сюръективному семейству $R(k)$. Строки $\vec{F}_{i_{1}, \ldots, i_{k-1}}^{f}$ и $\vec{F}_{l_{1}, \ldots, l_{k-1}}^{g}$ совпадают тогда и только тогда, когда сущчествует подстановка $\sigma \in S_{k-1}$, удовлетворяюшая условиям

1) $f\left(x_{1}, \ldots, x_{k-1}\right)=g\left(x_{\sigma(1)}, \ldots, x_{\sigma(k-1)}\right)$,

2) если $j_{1}<\cdots<j_{s}$ - номера всех существенных переменных функичи $f$, mo $i_{j_{1}}=l_{\sigma\left(j_{1}\right)}, \ldots, i_{j_{s}}=l_{\sigma\left(j_{s}\right)}$,

3) $\sigma\left(j_{1}\right)<\sigma\left(j_{2}\right)<\cdots<\sigma\left(j_{s}\right)$.

Доказательство. При доказательстве утверждения будем использовать следующее обозначение. Если у булевой функции $\varphi\left(x_{1}, \ldots, x_{k-1}\right)$ все существенные переменные есть $x_{\lambda_{1}}, \ldots, x_{\lambda_{s}}, \lambda_{1}<\cdots<\lambda_{s}$, то будем обозначать символом $\widetilde{\varphi}$ следующую булеву функцию от $s$ переменных $z_{1}, \ldots, z_{s}$ :

$$
\widetilde{\varphi}\left(z_{1}, \ldots, z_{s}\right)=f\left(0 \ldots 0 \underset{\lambda_{1} \text {-я координата }}{z_{1}} 0 \ldots 0 \underset{\lambda_{2} \text {-я координата }}{z_{2}} 0 \ldots 0 \underset{\lambda_{s} \text {-я координата }}{z_{s}} 0\right) .
$$

Очевидно, что $\varphi\left(\alpha_{1}, \ldots, \alpha_{k-1}\right)=\widetilde{\varphi}\left(\alpha_{j_{1}}, \alpha_{j_{2}}, \ldots, \alpha_{j_{s}}\right)$ для любого набора $\left(\alpha_{1}, \ldots, \alpha_{k-1}\right) \in V_{k-1}$.

Пусть строки $\vec{F}_{i_{1}, \ldots, i_{k-1}}^{f}$ и $\vec{F}_{l_{1}, \ldots, l_{k-1}}^{g}$ совпадают и пусть $j_{1}<\cdots<j_{s}-$ номера всех существенных переменных функции $f\left(x_{1}, \ldots, x_{k-1}\right)$. Тогда список номеров всех существенных переменных функции $F_{i_{1}, \ldots, i_{k-1}}^{f}\left(y_{1}, \ldots, y_{r}\right)=$ $f\left(y_{i_{1}}, \ldots, y_{i_{k-1}}\right)$ имеет вид $\left\{i_{j_{1}}, \ldots, i_{j_{s}}\right\}$. Пусть, далее, $t_{1}<\cdots<t_{s^{\prime}}-$ номера всех существенных переменных функции $g\left(x_{1}, \ldots, x_{k-1}\right)$. Тогда, аналогично, $\left\{l_{t_{1}}, \ldots, l_{t_{s^{\prime}}}\right\}$ - список номеров всех существенных переменных функции $F_{l_{1}, \ldots, l_{k-1}}^{g}\left(y_{1}, \ldots, y_{r}\right)=g\left(y_{l_{1}}, \ldots, y_{l_{k-1}}\right)$. Поскольку строки $\vec{F}_{i_{1}, \ldots, i_{k-1}}^{f}$ и $\vec{F}_{l_{1}, \ldots, l_{k-1}}^{g}$ совпадают, постольку совпадают и отображения $F_{i_{1}, \ldots, i_{k-1}}^{f}$ и $F_{l_{1}, \ldots, l_{k-1}}^{g}$, а значит, и списки номеров их существенных переменных. Поэтому $s=s^{\prime}$ и $\left\{l_{t_{1}}, \ldots, l_{t_{s^{\prime}}}\right\}=\left\{i_{j_{1}}, \ldots, i_{j_{s}}\right\}$. Так как $j_{1}<\cdots<j_{s}$ и $i_{1}<\cdots<i_{k-1}$, то $i_{j_{1}}<\cdots<i_{j_{s}}$. Аналогично $l_{t_{1}}<\cdots<l_{t_{s}}$. Следовательно, $i_{j_{1}}=l_{t_{1}}, \ldots, i_{j_{s}}=l_{t_{s}}$. 
Рассмотрим подстановку

$$
\sigma=\left(j_{1}, t_{1}\right)\left(j_{2}, t_{2}\right) \cdots\left(j_{s}, t_{s}\right) \in S_{k-1}
$$

заданную в виде произведений циклов длин 2 и 1 (единичные циклы не пишем).

Тогда, очевидно, $i_{j_{1}}=l_{\sigma\left(j_{1}\right)}, \ldots, i_{j_{s}}=l_{\sigma\left(j_{s}\right)}$ и $\sigma\left(j_{1}\right)<\sigma\left(j_{2}\right)<\cdots<\sigma\left(j_{s}\right)$.

Рассмотрим функцию $h\left(x_{1}, \ldots, x_{k-1}\right)=g\left(x_{\sigma(1)}, \ldots, x_{\sigma(k-1)}\right)$ и докажем, что функции $h$ и $f$ совпадают. Сначала установим, что совпадают функции $\widetilde{f}\left(z_{1}, \ldots, z_{s}\right)$ и $\widetilde{g}\left(z_{1}, \ldots, z_{s}\right)$.

Рассмотрим произвольный вектор $\left(\alpha_{1}, \ldots, \alpha_{s}\right) \in V_{s}$. Учитывая, что $i_{j_{1}}=$ $l_{t_{1}}, \ldots, i_{j_{s}}=l_{t_{s}}$, имеем

$$
\begin{aligned}
& \widetilde{g}\left(\alpha_{1}, \ldots, \alpha_{s}\right)= \\
& =g(\overbrace{0 \ldots 0 \underset{t_{1} \text {-я координата }}{\alpha_{1}} 0 \ldots 0 \underset{t_{s} \text {-я координата }}{\alpha_{s}} 0 \ldots 0}^{k-1 \text { кординат }})=
\end{aligned}
$$

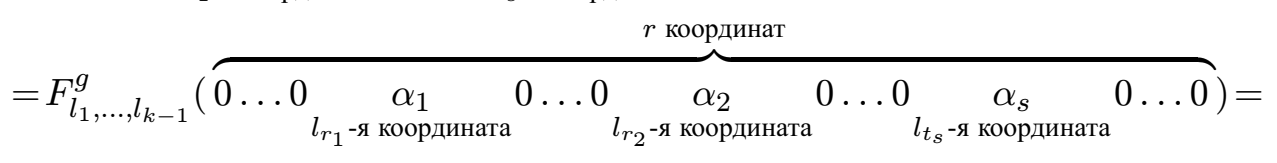

$$
\begin{aligned}
& r \text { координат } \\
& =F_{i_{1}, \ldots, i_{k-1}}^{f}(\overbrace{0 \ldots 0 \underset{l_{r_{1}} \text {-я координата }}{\alpha_{1}} 0 \ldots 0 \underset{l_{r_{2}} \text {-я координата }}{\alpha_{2}} 0 \ldots 0 \underset{l_{t_{s}} \text {-я координата }}{\alpha_{s}} 0 \ldots 0}^{\alpha_{s}})= \\
& r \text { координат } \\
& =F_{i_{1}, \ldots, i_{k-1}}^{f}(\overbrace{0 \ldots 0 \underset{i_{j_{1}} \text {-я координата }}{\alpha_{1}} 0 \ldots 0 \underset{i_{j_{2}} \text {-я координата }}{\alpha_{2}} 0 \ldots 0 \underset{i_{j_{s}} \text {-я координата }}{\alpha_{s}} 0 \ldots 0}^{\alpha_{s}})= \\
& k-1 \text { координат }
\end{aligned}
$$

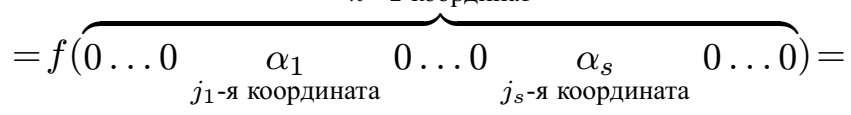

$$
\begin{aligned}
& =\tilde{f}\left(\alpha_{1}, \ldots, \alpha_{s}\right) \text {. }
\end{aligned}
$$

Теперь докажем, что совпадают функции $f$ и $h$. Пусть $\left(\beta_{1}, \ldots, \beta_{k-1}\right) \in V_{k-1}$ есть произвольный вектор. Учитывая, что $\sigma\left(t_{1}\right)=j_{1}, \ldots, \sigma\left(t_{s}\right)=j_{s}$, имеем

$$
\begin{aligned}
& h\left(\beta_{1}, \ldots, \beta_{k-1}\right)= \\
& =g\left(\beta_{\sigma(1)}, \ldots, \beta_{\sigma(k-1)}\right)=\widetilde{g}\left(\beta_{\sigma\left(t_{1}\right)}, \ldots, \beta_{\sigma\left(t_{s}\right)}\right)= \\
& =\widetilde{f}\left(\beta_{\sigma\left(t_{1}\right)}, \ldots, \beta_{\sigma\left(t_{s}\right)}\right)=\widetilde{f}\left(\beta_{j_{1}}, \ldots, \beta_{j_{s}}\right)= \\
& =f\left(\beta_{1}, \ldots, \beta_{k-1}\right) .
\end{aligned}
$$


В другую сторону: пусть $x_{j_{1}}, \ldots, x_{j_{s}}, j_{1}<\cdots<j_{s}$, - это все существенные переменные функции $f\left(x_{1}, \ldots, x_{k-1}\right)$. Пусть существует $\sigma \in S_{k-1}$, удовлетворяющая условиям 1)-3). Тогда все номера существенных переменных функции $g$ есть $\sigma\left(j_{1}\right), \ldots, \sigma\left(j_{s}\right)$. Рассмотрим произвольный вектор $\left(\gamma_{1}, \ldots, \gamma_{r}\right) \in V_{r}$. Имеем

$$
\begin{aligned}
& F_{i_{1}, \ldots, i_{k-1}}^{f}\left(\gamma_{1}, \ldots, \gamma_{r}\right)= \\
& =f\left(\gamma_{i_{1}}, \ldots, \gamma_{i_{k-1}}\right)=\tilde{f}\left(\gamma_{i_{1}}, \ldots, \gamma_{i_{j_{s}}}\right)= \\
& k-1 \text { координат }
\end{aligned}
$$

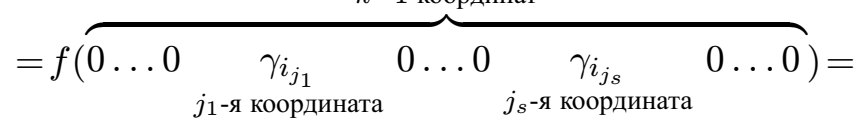

$$
\begin{aligned}
& =g\left(0 \ldots 0 \underset{\sigma\left(j_{1}\right) \text {-я координата }}{\gamma_{i_{j_{1}}}} \quad 0 \ldots 0 \underset{\sigma\left(j_{s}\right) \text {-я координата }}{\gamma_{i_{j_{s}}}} \quad 0 \ldots 0\right)= \\
& =g\left(0 \ldots 0 \underset{\sigma\left(j_{1}\right) \text {-я координата }}{\gamma_{l_{\sigma}\left(j_{1}\right)}} \quad 0 \ldots 0 \underset{\sigma\left(j_{s}\right) \text {-я координата }}{\gamma_{l_{\sigma}\left(j_{s}\right)}} 0 \ldots 0\right)=g\left(\gamma_{l_{1}}, \ldots, \gamma_{l_{k-1}}\right)= \\
& =F_{l_{1}, \ldots, l_{k-1}}^{g}\left(\gamma_{1}, \ldots, \gamma_{r}\right) \text {. }
\end{aligned}
$$

Следовательно, строки $\vec{F}_{i_{1}, \ldots, i_{k-1}}^{f}$ и $\vec{F}_{l_{1}, \ldots, l_{k-1}}^{g}$ совпадают.

Вообще говоря, использование доказанного критерия наличия совпадающих строк в матрице $M\langle R(k, r)\rangle$ для вычисления их числа представляется достаточно сложным делом, поскольку требуется знание группы инерции каждой булевой функции из $k$-сюръективного семейства $R(k, r)$. Далее рассмотрим несколько случаев, когда такое вычисление удается выполнить за счет простоты структуры или небольшого размера семейства $R(k, r)$.

Получим несколько следствий из теоремы 3.

Следствие 1. Пусть матрица $M\langle R(k, r)\rangle$ построена по $k$-сюръективному семейству $R(k)$ и функиия $f\left(x_{1}, \ldots, x_{k-1}\right)$ из этого семейства имеет существенные переменные $x_{j_{1}}, \ldots, x_{j_{s}}, j_{1}<\cdots<j_{s}$. Тогда строки $\vec{F}_{i_{1}, \ldots, i_{k-1}}^{f} u$ $\vec{F}_{l_{1}, \ldots, l_{k-1}}^{f}$ совпадают тогда и только тогда, когда $i_{j_{1}}=l_{j_{1}}, \ldots, i_{j_{s}}=l_{j_{s}}$.

Доказательство. Согласно теореме 3 совпадение строк $\vec{F}_{i_{1}, \ldots, i_{k-1}}^{f}$ и $\vec{F}_{l_{1}, \ldots, l_{k-1}}^{f}$ эквивалентно существованию подстановки $\sigma \in S_{k-1}$, удовлетворяющей условиям

1) $f\left(x_{1}, \ldots, x_{k-1}\right)=f\left(x_{\sigma(1)}, \ldots, x_{\sigma(k-1)}\right)$,

2) $i_{j_{1}}=l_{\sigma\left(j_{1}\right)}, \ldots, i_{j_{s}}=l_{\sigma\left(j_{s}\right)}$,

3) $\sigma\left(j_{1}\right)<\sigma\left(j_{2}\right)<\cdots<\sigma\left(j_{s}\right)$. 
Из условия 1) получаем, что существенные переменные функции $f$ имеют номера $\left\{\sigma\left(j_{1}\right), \ldots, \sigma\left(j_{s}\right)\right\}$, т. е. $\left\{\sigma\left(j_{1}\right), \ldots, \sigma\left(j_{s}\right)\right\}=\left\{j_{1}, \ldots, j_{s}\right\}$. Так как $j_{1}<$ $\cdots<j_{s}$ и $\sigma\left(j_{1}\right)<\cdots<\sigma\left(j_{s}\right)$, то $\sigma\left(j_{1}\right)=j_{1}, \ldots, \sigma\left(j_{s}\right)=j_{s}$.

В другую сторону: если $i_{j_{1}}=l_{j_{1}}, \ldots, i_{j_{s}}=l_{s}$, то любая подстановка $\sigma \in S_{k-1}$, для которой $\sigma\left(j_{1}\right)=j_{1}, \ldots, \sigma\left(j_{s}\right)=j_{s}$, очевидно, удовлетворяет условиям 1)-3).

Следствие 2. Если функция $f\left(x_{1}, \ldots, x_{k-1}\right)$ из $k$-сюръективного семейства $R(k)$ существенно зависит от всех своих переменных, то в матрице $M\langle R(k, r)\rangle$ нет совпадающих строк вида $\vec{F}_{i_{1}, \ldots, i_{k-1}}^{f} u \vec{F}_{l_{1}, \ldots, l_{k-1}}^{f}$.

Доказательство. По следствию 1 если строки $\vec{F}_{i_{1}, \ldots, i_{k-1}}^{f}$ и $\vec{F}_{l_{1}, \ldots, l_{k-1}}^{f}$ совпадают, то $i_{1}=l_{1}, \ldots i_{k-1}=l_{k-1}$.

Рассмотрим 2-сюръективное семейство

$$
T(2)=\left\{f_{1}=x_{1}, f_{2}=x_{1} \oplus 1, f_{3}=0, f_{4}=1\right\}, \quad r \geqslant k=2 .
$$

Очевидно, что ни одна из функций $f_{1}, f_{2}, f_{3}, f_{4}$ в другую перестановкой переменных не переводится, поэтому по теореме 3 в $M\langle R(k, r)\rangle$ пары строк $\vec{F}_{i_{1}}^{f_{v}}, \quad \vec{F}_{l_{1}}^{f_{w}}$ всегда различны при $v \neq w, v, w \in\{1,2,3,4\}$. Функции $f_{1}$, $f_{2}$ существенно зависят от всех своих переменных, поэтому воспользуемся следствием 2 и получим, что строки $\vec{F}_{i_{1}}^{f_{v}}, \vec{F}_{l_{1}}^{f_{v}}$ также различны при $i \neq l_{1}$, $1 \leqslant i_{1}, l_{1} \leqslant r, \quad v \in\{1,2,3,4\}$. Очевидно, что все строки $\vec{F}_{i_{1}}^{f_{3}}\left(1 \leqslant i_{1} \leqslant r\right)$ есть строки из одних нулей, а строки $\vec{F}_{i_{1}}^{f_{4}}\left(1 \leqslant i_{1} \leqslant r\right)-$ строки из одних единиц. Таким образом, в матрице $M\langle T(2, r)\rangle$ число различных строк вида $\vec{F}_{i_{1}}^{f_{1}}$ равно $r$, строк вида $\vec{F}_{i_{1}}^{f_{2}}$ тоже $r$, различных строк вида $\vec{F}_{i_{1}}^{f_{3}}$ только одна и вида $\vec{F}_{i_{1}}^{f_{4}}$ также одна. Тем самым доказано следующее утверждение.

Утверждение 6. Число различных строк в матрице $M\langle T(2, r)\rangle$ равно $2 r+2$.

Для сравнения приведем значение $n_{\max }^{(2)}(m)$ максимального числа столбцов в комбинаторно полной матрице порядка 2 с $m$ строками, найденное в [1]. При четном $m$ верно равенство $n_{\max }^{(2)}(m)=\frac{1}{2} \cdot\left(\begin{array}{c}m \\ m / 2\end{array}\right)$. Используя при $m \geqslant 6$ элементарные оценки $2^{m / 2}<\left(\begin{array}{c}m \\ m / 2\end{array}\right)<2^{m}$, получаем $2^{r}<n_{\max }^{(2)}(2 r+2)<$ $2^{2 r+1}$. В матрице $M\langle T(2, r)\rangle$ согласно утверждению 6 имеется $2 r+2$ строк и $2^{r}$ столбцов. 
Перейдем к 3-сюръективному семейству

$$
\begin{aligned}
T(3)=\left\{f_{1}=\right. & x_{1} \oplus x_{2}, f_{2}=\overline{x_{1} \oplus x_{2}}, f_{3}=x_{1}, f_{4}=\overline{x_{1}}, \\
& \left.f_{5}=x_{2}, f_{6}=\overline{x_{2}}, f_{7}=0, f_{8}=1\right\}, \quad r \geqslant k=3 .
\end{aligned}
$$

Все семейство разбивается на следующие классы эквивалентности относительно перестановок переменных:

$$
T(3)=\left\{f_{1}\right\} \cup\left\{f_{2}\right\} \cup\left\{f_{3}, f_{5}\right\} \cup\left\{f_{4}, f_{6}\right\} \cup\left\{f_{7}\right\} \cup\left\{f_{8}\right\} .
$$

Функции $f_{1}$ и $f_{2}$ существенно зависят от всех своих переменных, поэтому по следствию 2 не дают совпадающих строк вида $\vec{F}_{i_{1}, i_{2}}^{f_{v}}, \vec{F}_{l_{1}, l_{2}}^{f_{v}}$ при $\left\{i_{1}, i_{2}\right\} \neq\left\{l_{1}, l_{2}\right\}, 1 \leqslant i_{1} \leqslant i_{2} \leqslant r, 1 \leqslant l_{1}<l_{2} \leqslant r, v \in\{1,2\}$.

У функции $f_{3}$ существенная переменная только $x_{1}$, т. е. по следствию 1 с $s=1, j_{1}=1$ равенство строк $\vec{F}_{i_{1}, i_{2}}^{f_{3}}=\vec{F}_{l_{1}, l_{2}}^{f_{3}}$ имеет место тогда и только тогда, когда $i_{1}=l_{1}$. Значит, из всего набора строк $\left\{\vec{F}_{i_{1}, i_{2}}^{f_{3}}, 1 \leqslant i_{1} \leqslant i_{2} \leqslant r\right\}$ наибольшее подмножество из различных строк определяется наибольшим количеством пар $\left(i_{1}, i_{2}\right), 1 \leqslant i_{1}<i_{2} \leqslant r$, с различными первыми координатами. Очевидно, что число таких пар равно $r-1$, например $\{(1,2),(2,3), \ldots$ $\ldots,(r-1, r)\}$.

Аналогично доказываем, что среди всех строк $\left\{\vec{F}_{i_{1}, i_{2}}^{f_{4}}, 1 \leqslant i_{1}<i_{2} \leqslant r\right\}$ только $r-1$ различных.

Перейдем к функции $f_{5}\left(x_{1}, x_{2}\right)=x_{2}$. В условиях теоремы 3 в качестве функции $f$ рассмотрим функцию $f_{3}\left(x_{1}, x_{2}\right)=x_{1}$, тогда $s=1, j_{1}=1$, а в качестве функции $g-$ функцию $f_{5}$, тогда существует такая подстановка $\sigma=\left(\begin{array}{ll}1 & 2 \\ 2 & 1\end{array}\right)$, что $f\left(x_{1}, x_{2}\right)=g\left(x_{\sigma(1)}, x_{\sigma(2)}\right)$. По теореме 3 строка $\vec{F}_{i_{1}, i_{2}}^{f_{3}}, 1 \leqslant$ $i_{1}<i_{2} \leqslant r$, совпадет со строкой $\vec{F}_{l_{1}, l_{2}}^{f_{5}}, 1 \leqslant l_{1}<l_{2} \leqslant r$, тогда и только тогда, когда $i_{1}=l_{\sigma(1)}=l_{2}$. Значит, каждая строка $\vec{F}_{l_{1}, l_{2}}^{f_{5}}, \quad 1 \leqslant l_{1}<l_{2}<r$, совпадает со строкой $\vec{F}_{l_{2}, i_{2}}^{f_{3}}$ и строки $\vec{F}_{l_{1}, r}^{f_{5}}, \quad 1 \leqslant l_{1} \leqslant r-1$, не имеют повторов среди строк $\vec{F}_{i_{1}, i_{2}}^{f_{3}}, 1 \leqslant i_{1}<i_{2} \leqslant r$. Далее, рассмотрим две строки $\vec{F}_{i_{1}, r}^{f_{5}}, \quad \vec{F}_{l_{1}, r}^{f_{5}}, \quad 1 \leqslant i_{1}, \quad l_{1} \leqslant r-1$. У функции $f_{5}$ существенная переменная только одна $-x_{2}$, и по следствию 1 эти строки совпадают. Следовательно, в матрице $M\langle T(3, r)\rangle$, удаляя повторы, можно оставить только одну строку, соответствующую функции $f_{5}$, а именно строку $\vec{F}_{1, r}^{f_{5}}$.

Аналогично доказывается, что для функции $f_{6}$ можно оставить только одну строку $\vec{F}_{1, r}^{f_{6}}$.

Строки, соответствующие функциям $f_{7}$ и $f_{8}$, состоят из одних нулей или одних единиц и поэтому дают только две различные строки. 
Таким образом, множество различных строк в матрице $M\langle T(3, r)\rangle$ можно описать следующим образом:

$\left(\begin{array}{l}r \\ 2\end{array}\right)$ строк вида $\vec{F}_{i_{1}, i_{2}}^{f_{1}}\left(1 \leqslant i_{1}<i_{2} \leqslant r\right)$,

$\left(\begin{array}{l}r \\ 2\end{array}\right)$ строк вида $\vec{F}_{i_{1}, i_{2}}^{f_{2}}\left(1 \leqslant i_{1}<i_{2} \leqslant r\right)$,

$r-1$ строк вида ${ }_{1,2}^{f_{3}}, \vec{F}_{1,2}^{f_{3}}, \vec{F}_{2,3}^{f_{3}}, \ldots, \vec{F}_{r-1, r}^{f_{3}}$,

$r-1$ строк вида $\vec{F}_{1,2}^{f_{4}}, \vec{F}_{2,3}^{f_{4}}, \ldots, \vec{F}_{r-1, r}^{f_{4}}$,

по одной строке вида $\vec{F}_{1, r}^{f_{5}}, \vec{F}_{1, r}^{f_{6}}, \vec{F}_{1,2}^{f_{6}}, \vec{F}_{1,2}^{f_{7}}$.

Сформулируем полученный результат в виде утверждения 7.

Утверждение 7. Число различных строк в матрице $M\langle T(3, r)\rangle$ равно $2 \cdot\left(\left(\begin{array}{l}r \\ 2\end{array}\right)+r+1\right)$.

Известные оценки для $m_{\min }^{(k)}(n)$ (минимально возможного числа строк комбинаторно полной матрицы порядка $k$ с $n$ столбцами), указанные во введении, при $k=3$ принимают вид $17 \cdot \log _{2} n>m_{\min }^{(3)}(n)>2 \cdot \log _{2} n$ для достаточно больших значений $n$. Если обозначить число столбцов матрицы $M\langle T(3, r)\rangle$, равное $2^{r}$, через $n$, то число строк этой матрицы будет $2 \cdot\left(\left(\begin{array}{c}\log _{2} n \\ 2\end{array}\right)+\log _{2} n+1\right)=\left(\log _{2} n\right)^{2}+\log _{2} n+2$.

Отметим простой случай в виде еще одного следствия теоремы 3.

Следствие 3. Если в $k$-сюръективном семействе $R(k)$ все функиии не имеют несущественных переменных, то в матрице $M\langle R(k, r)\rangle$ нет совпадающих строк.

Доказательство. Пусть функции $f, g \in R(k)$ зависят от всех своих переменных и строки $\vec{F}_{i_{1}, \ldots, i_{k-1}}^{f}, \vec{F}_{l_{1}, \ldots, l_{k-1}}^{g}$ совпадают. По теореме 3 существует такая перестановка $\sigma \in S_{k-1}$, что $f\left(x_{1}, \ldots, x_{k-1}\right)=g\left(x_{\sigma(1)}, \ldots, x_{\sigma(k-1)}\right)$, $i_{1}=l_{\sigma(1)}, \ldots, i_{k-1}=l_{\sigma(k-1)}$ и $\sigma(1)<\sigma(2)<\ldots<\sigma(k-1)$. Но последнее возможно только в случае, когда $\sigma(1)=1, \ldots, \sigma(k-1)=k-1$, и тогда $f\left(x_{1}, \ldots, x_{k-1}\right)=g\left(x_{1}, \ldots, x_{k-1}\right), i_{1}=l_{1}, \ldots, i_{k-1}=l_{k-1}$. Значит, $\vec{F}_{i_{1}, \ldots, i_{k-1}}^{f}$ и $\vec{F}_{l_{1}, \ldots, l_{k-1}}^{g}-$ это одна и та же строка матрицы $M\langle R(k, r)\rangle$.

Рассмотрим семейство $E(k), \quad k \geqslant 4$. Напомним, что $|E(k)|=\left(\begin{array}{c}2^{k-1} \\ k\end{array}\right)$. Из определения семейства следует, что все функции в этом семействе имеют один и тот же вес, равный $k$. При нечетном значении $k$ вес функций также нечетен, а функции нечетного веса не имеют несущественных переменных.

Утверждение 8. При нечетном значении $k \geqslant 5$ в матрице $M\langle E(k, r)\rangle$ нет совпадающих строк и число строк в этой матрице равно $\left(\begin{array}{c}r \\ k-1\end{array}\right) \cdot\left(\begin{array}{c}2^{k-1} \\ k\end{array}\right)$.

Утверждение 8 вытекает из следствия 3. 


\section{Список литературы}

[1] Ролдугин П. В., Тарасов А. В., “О булевых функциях без верхних биюнктивных статаналогов”, Математические проблемы криптографии, 4:1 (2013), 123-140.

[2] Ролдугин П. В., Тарасов А. В., “Функции без коротких имплицент. Часть I: нижние оценки весов”, Дискретная математика, $27: 2$ (2015), 94-105.

[3] Ролдугин П. В., Тарасов А.В., “Функции без коротких имплицент. Часть II: методы построения”, Дискретная математика, $27: 4$ (2015), 120-132.

[4] Райгородский А. М., Системы общих представителей и их приложения в геометрии, М.: МЦНМО, 2009, 136 pp.

[5] Логачев О.А., Сальников А. А., Ященко В.В., Булевы функиии в теории кодирования и криптологии, М.: МЦНМО, 2004, 470 рр.

[6] Глухов М. М., Шишков А. Б., Математическая логика. Дискретные функции. Теория алгоритмов, СПб: Лань, 2012, 400 pp.

[7] Биркгоф Г., Теория решеток, М.: Наука, 1984, 568 рp.

[8] Сачков В. Н., Тараканов В. Е., Комбинаторика неотрицательных матрии, М.: ТВП, 2000, $448 \mathrm{pp}$. 